\title{
NOUVELLE
}

\section{Le système nerveux autonome}

\section{Un nouveau composant du \\ microenvironnement tumoral}

Claire Magnon ${ }^{1,2,3}$
${ }^{1}$ Ruth L. and David S. Gottesman Institute for Stem Cell and Regenerative Medicine Research, Departments of ${ }^{2}$ Medicine and ${ }^{3}$ Cell Biology. Albert Einstein College of Medicine, 1300 Morris Park Avenue, Bronx, New York, NY10460, États-Unis.

clairemagnon@free.fr
> Le cancer se caractérise par une extrême hétérogénéité phénotypique liée, en partie, à la diversité génétique ou épigénétique des différentes populations de cellules tumorales qui le composent [1]. L'émergence de cellules cancéreuses, due à l'acquisition de mutations ou à l'altération de l'expression de certains gènes, est un événement assez fréquent au cours de la vie d'un sujet, qui reste asymptomatique dans la majorité des cas [2]. La formation de tumeurs malignes est plus rarement observée et nécessite le concours du stroma [2]. En effet, l'étude du microenvironnement tumoral a montré que les altérations du stroma environnant les cellules cancéreuses sont des facteurs de sélection majeurs, qui participent activement à l'élaboration d'une tumeur et contribuent également à son hétérogénéité [2, 3].

\section{Le microenvironnement tumoral :}

\section{un état des lieux}

Le microenvironnement comprend une variété de cellules et de composants non cellulaires (matrice extracellulaire) qui interagissent lors de la construction d'un organe en contribuant à la différenciation guidée des cellules souches et des progéniteurs durant le développement embryonnaire [2]. De façon similaire, le microenvironnement interagit avec les cellules cancéreuses et, dans certaines circonstances, est détourné de sa fonction physiologique première à la faveur du développement tumoral et de sa progression. Le stroma «pathologique » qui en résulte comprend, entre autres, des cellules endothéliales et lymphatiques, des péricytes, des fibroblastes, des cellules dérivées de la moelle osseuse et des métalloprotéinases matricielles. Le stroma subvient à l'apport de nutriments et d'oxygène grâce au développement d'un système vasculaire tumoral, mais participe aussi à la formation d'une immunité protumorale, ainsi qu'à la rupture de la membrane basale, concourant ainsi à la dissémination des cellules cancéreuses (Figure 1). La composition du microenvironnement est très hétérogène selon les différents types de cancer, et même au sein d'un même type de tumeur. Cette hétérogénéité est directement corrélée à son activité sur le comportement des cellules cancéreuses, et influence et contraint le développement d'une tumeur jusqu'à sa progression.

Récemment, nous avons montré que le microenvironnement tumoral comprend également des fibres nerveuses issues du système nerveux autonome (SNA), qui contribuent activement à l'initiation d'une tumeur et à sa progression [4].

Le système nerveux sympathique: un prérequis pour le développement initial du cancer

Le système nerveux autonome, composé des systèmes nerveux sympathique (SNS) et parasympathique (PNS), est connu pour sa participation à l'homéostasie des organes périphériques. En particulier, la prostate est richement innervée par les deux branches du SNA qui régulent la croissance et la régénération de la glande [5]. Des études ont montré que, selon un processus nommé invasion périneurale, certains cancers épithéliaux de mauvais pronostic, dont le cancer de la prostate, sont entourés de larges nerfs envahis de cellules tumorales, suggérant une nouvelle voie de dissémination métastatique [6]. II a été également montré que l'activation des voies de signalisation adrénergique, induite par des conditions expérimentales de stress dans des modèles murins, favorise le développement tumoral et le processus métastatique [7-9]. Le neurotransmetteur noradrénaline (NA) activerait alors le récepteur adrénergique $\beta 2$ (Adrb2) exprimé à la surface de cellules tumorales ovariennes ou mammaires et induirait ainsi prolifération et croissance tumorales [9].

Nous avons récemment montré par imagerie bioluminescente et microscopie confocale que les tumeurs de la prostate sont infiltrées de fibres nerveuses sympathiques qui contrôlent les phases précoces du développement tumoral [4]. Lorsque des sympathectomies chimique ou chirurgicale de la prostate sont réalisées, la prise de greffe orthotopique de cellules cancéreuses prostatiques ( $P C$-3luc qui expriment le gène rapporteur luciférase) est enrayée ; il en est de même du développement des lésions néoplasiques intraépithéliales (prostate intraepithelial neoplasia, PIN) dans un modèle murin transgénique qui surexprime le protooncogène $\mathrm{c}-\mathrm{Myc}$ ( $\mathrm{Hi}-\mathrm{Myc}$ ) sous le contrôle du promoteur probasin (un promoteur spécifique de la prostate). La destruction des fibres nerveuses sympathiques entraîne alors l'apoptose des cellules néoplasiques prostatiques. Ces résultats ont été confirmés par délétion génétique des récepteurs adrénergiques $\beta 2(\operatorname{Adr} \beta 2)$ et $\beta 3(\operatorname{Adr} \beta 3)$ exprimés par 


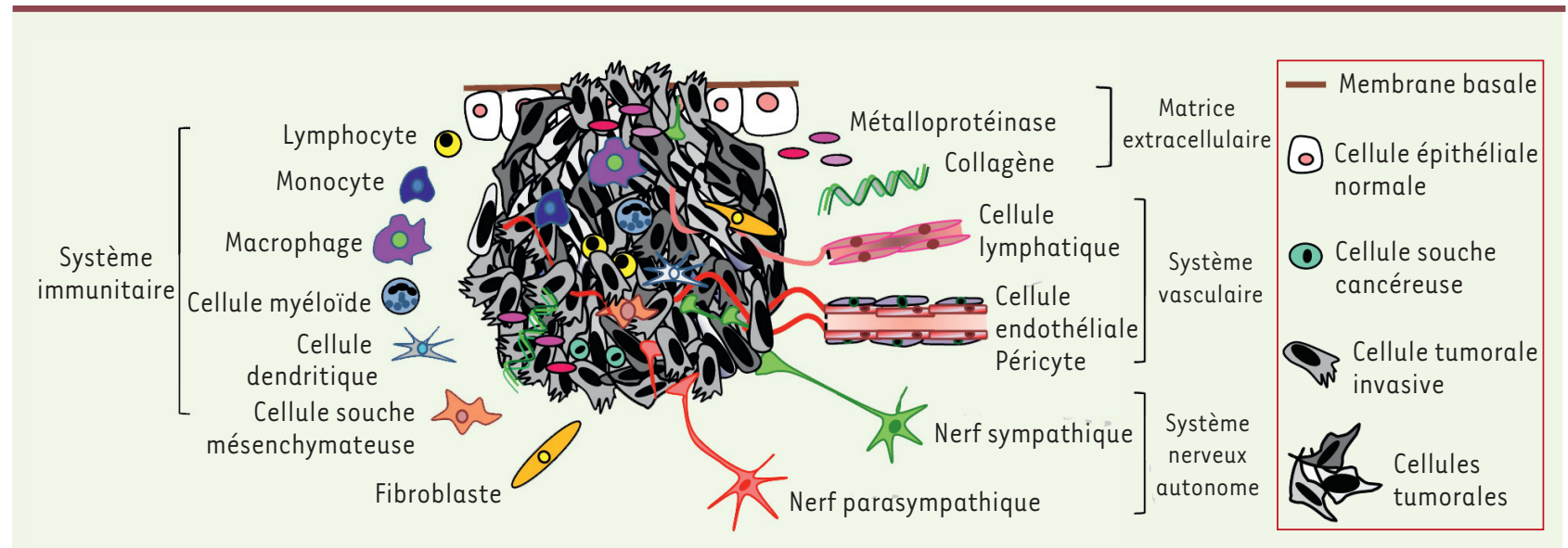

Figure 1. L’hétérogénéité tumorale. La tumeur primaire est composée de cellules cancéreuses phénotypiquement différentes dues à des altérations génétiques et épigénétiques. À cette diversité s'ajoute un microenvironnement tumoral complexe incluant des cellules dérivées de la moelle osseuse (bone marrow derived-cells, BMDC) dont les macrophages tumoraux (tumor-associated macrophages, TAM) ; les cellules suppressives dérivées des cellules myéloïdes (myeloid cell-derived suppressor cells, MDSC); les cellules souches mésenchymateuses (mesenchymal stem cells, MSC) ; les monocytes exprimant Tie2 (Tie2-expressing monocytes, TEM) ; mais aussi des fibroblastes, des cellules endothéliales et lymphatiques, des composants non cellulaires et des fibres nerveuses autonomes qui participent activement à la survie des cellules cancéreuses, à leur prolifération ainsi qu’à leur dissémination.

le stroma prostatique, et confirme le rôle du SNS dans le contrôle de l'initiation tumorale dans la prostate.

Le système nerveux parasympathique : un régulateur de la dissémination tumorale

Nous avons observé que les tumeurs prostatiques sont également infiltrées par des fibres nerveuses cholinergiques parasympathiques dont la présence corrèle avec le caractère métastatique de la tumeur [4]. L'administration d'un agoniste non sélectif (carbachol) des récepteurs muscariniques aux souris xénogreffées avec les cellules tumorales $P C$-3luc a révélé une augmentation significative de la dissémination des cellules cancéreuses dans les ganglions lymphatiques entourant la prostate et dans les organes périphériques. L'adjonction d'un antagoniste non sélectif (scopolamine), ou sélectif (pirenzepine), du récepteur muscarinique de type 1 (Chrml) a significativement enrayé l'activité invasive et métastatique du carbachol. Des études pharmacologiques similaires effectuées dans le modèle transgénique $\mathrm{Hi}$ -
Myc ont confirmé le rôle pro-invasif et prométastatique des fibres nerveuses cholinergiques parasympathiques via le récepteur Chrml. De plus, lorsque les expériences de xénogreffes de cellules tumorales ont été réalisées chez des animaux génétiquement déficients en Chrml, les résultats ont corroboré ceux des études pharmacologiques conduites chez des animaux non génétiquement modifiés. Ces deux types d'études, pharmacologiques et génétiques, ont démontré que l'innervation cholinergique parasympathique régule spécifiquement les processus invasif et métastatique au sein de la tumeur primaire.

\section{Vers une nouvelle thérapie ciblée du cancer de la prostate?}

Ces dernières années ont vu l'émergence de nouvelles thérapeutiques, dites ciblées, des cancers. Parmi celles-ci, on peut citer le Bévacizumab (Avastin ${ }^{\circledR}$ ), un anticorps agissant sur le microenvironnement tumoral en bloquant le développement des vaisseaux infiltrant les tumeurs. II bloque sélectivement l'action du vascular endothelial growth factor (VEGF), un facteur angiogénique qui stimule le développement vasculaire. Si ces thérapies anti-angiogéniques ont apporté un certain bénéfice thérapeutique, de nouvelles résistances sont malheureusement apparues à l'arrêt de leur administration. Cependant, un intéressant parallèle peut être fait entre l'infiltration des vaisseaux et celle des nerfs au sein des tumeurs. Le cancer recrute et s'approprie, au cours de son développement, ces deux composants du stroma en les détournant de leur fonction physiologique à des fins pathologiques, créant ainsi une angiogenèse et une neurogenèse tumorales. De façon intéressante, de récentes études épidémiologiques, menées chez des patients développant des cancers de la prostate, du sein ou des mélanomes, ont récemment montré que l'administration de bêta-bloquants non sélectifs, tel que le propranolol, diminue l'incidence des cancers et le taux de mortalité [1012]. Ces études épidémiologiques corroborent nos études cliniques, menées sur une cohorte de patients développant un cancer de la prostate à bas ou à haut risque de progression tel que le définissent le score de Gleason, le taux de 


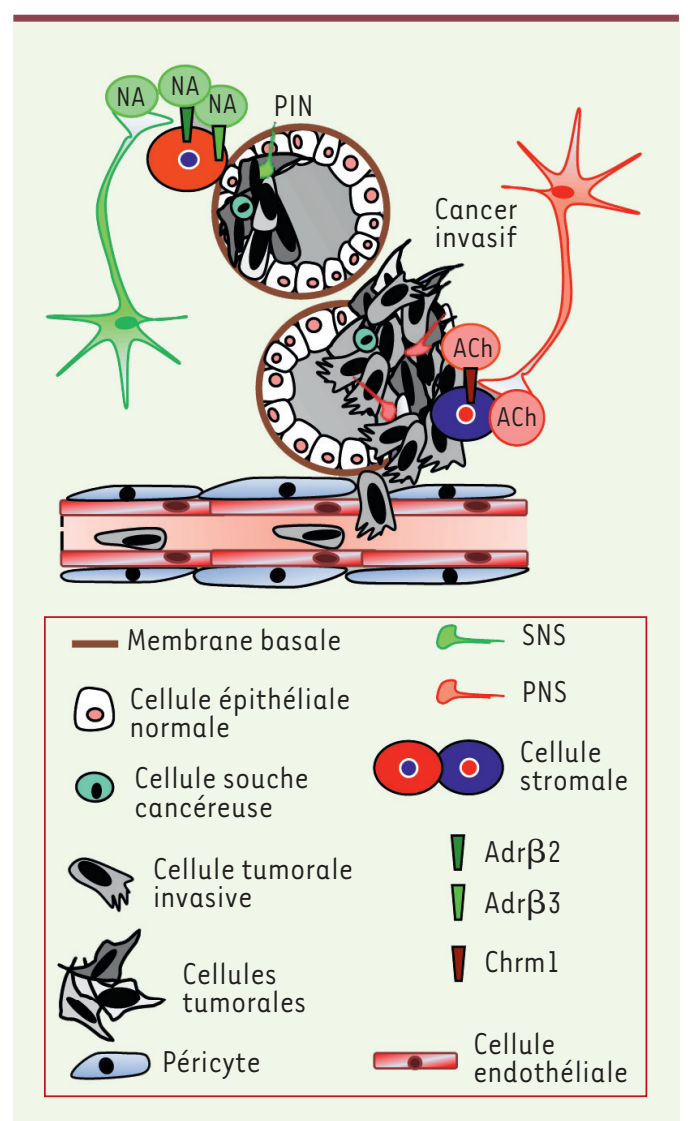

Figure 2. Le système nerveux autonome contribue au développement tumoral et à sa progression. Alors que le système nerveux sympathique (SNS) contrôle les phases initiales de la formation de la tumeur en activant les récepteurs adrénergiques b2 et b3 (Adr b2 et Adrb3) exprimés dans le stroma, le système nerveux parasympathique (PNS) stimule la dissémination métastatiques des cellules cancéreuses en agissant sur le récepteur muscarinique de type l (Chrml) présent dans le microenvironnement tumoral prostatique. NA : noradrénaline; Ach : acétylcholine; PIN : prostate intraepithelial neoplasia.

PSA et les stades histologique et clinique [4]. L'analyse de la densité des fibres nerveuses sympathiques et parasympathiques a montré une augmentation significative de l'infiltration nerveuse au sein des tumeurs à haut risque par comparaison aux tumeurs à bas risque de progression. Plus précisément, lorsque la densité de fibres nerveuses intratumorales et dans les tissus prostatiques normaux entourant la tumeur primaire est élevée, l'évolution tumorale est péjorative avec une extension extracapsulaire et un taux de récidive plus élevé [4]. L'administration de thérapeutiques ciblées, telles que des antagonistes sélectifs de $\operatorname{Adr} \beta 2$ et $\operatorname{Adr} \beta 3$ combinés ou non à un antagoniste sélectif de Chrml, permettrait alors de contrôler de façon spécifique les activités noradrénergique et cholinergique des fibres nerveuses tumorales, régulant, respectivement, tumorigenèse et métastases. À l'instar des thérapies ciblées anti-angiogéniques, il est aussi permis d'envisager le développement de nouvelles thérapies anti-neurogéniques bloquant l'activité de facteurs de croissance neuronale. Ceci permettrait peut-être aussi d'endiguer les résistances tumorales dues aux traitements antiangiogéniques du fait de l'intrication des mécanismes de développement des nerfs et des vaisseaux.

Notre étude, appuyée par deux modèles tumoraux murins et l'analyse rétrospective d'une cohorte de patients, identifie les activités spécifique et complémentaire des deux branches du SNA dans le développement tumoral de la prostate. Alors que le SNS contrôle l'initiation tumorale, le PNS contribue, quant à lui, aux processus invasif et métastatique. Cette dualité d'activité permet d'envisager le développement de thérapeutiques ciblant les voies de signalisation neuronale afin de combattre, simultanément et/ou spécifiquement, tumorigenèse et métastases. $\diamond$

Autonomic nerves in cancer: a missing piece of the jigsaw puzzle

\section{LIENS D'INTÉRÊT}

L'auteur déclare n'avoir aucun lien d'intérêt concernant les données publiées dans cet article.

\section{RÉFÉRENCES}

1. Marusyk A, Almendro V, Polyak K. Intra-tumour heterogeneity : a looking glass for cancer? Nat Rev Cancer 2012 ; 12 : 323-34.

2. Bissell MJ, Hines WC. Why don't we get more cancer? A proposed role of the microenvironment in restraining cancer progression. Nat Med $2011 ; 17$; 320-9.

3. Mintz B, Illmensee K. Normal genetically mosaic mice produced from malignant teratocarcinoma cells. Proc Natl Acad Sci USA 1975 ; 72 : 3585-9.

4. Magnon C, Hall SJ, Lin J, et al. Autonomic nerve development contributes to prostate cancer progression. Science 2013 ; 341 : 1236361

5. Pennefather JN, Lau WA, Mitchelson F, Ventura S. The autonomic and sensory innervation of the smooth muscle of the prostate gland: a review of pharmacological and histological studies. J Auton Pharmacol $2000 ; 20: 193-206$.

6. Liebig C, Ayala G, Wilks JA, et al. Perineural invasion in cancer : a review of the literature. Cancer 2009; 115 : 3379-91.

7. Simon RH, Lovett $\varepsilon$ J 3rd, Tomaszek D, Lundy J. Electrical stimulation of the midbrain mediates metastatic tumor growth. Science $1980 ; 209$ : 1132-3.

8. Visintainer MA, Volpicelli JR, Seligman ME. Tumor rejection in rats after inescapable or escapable shock. Science $1982 ; 216: 437-9$

9. Thaker PH, Han Ly, Kamat AA, et al. Chronic stress promotes tumor growth and angiogenesis in a mouse model of ovarian carcinoma. Nat Med 2006 ; 12 : 939 44.

10. Grytli HH, Fagerland MW, Fossa SD, Tasken KA. Association between use of beta-blockers and prostate cancer-specific survival : A cohort study of 3561 prostate cancer patients with high-risk or metastatic disease. Eur Urol 2013 (sous presse).

11. Barron TI, Connolly RM, Sharp L, et al. Beta blockers and breast cancer mortality : a population- based study. I Clin Oncol 2011 ; 29 : 2635-44

12. Lemeshow S, Sorensen HT, Phillips G, et al. Betablockers and survival among Danish patients with malignant melanoma : a population-based cohort study. Cancer Epidem Biomark 2011 ; 20 : 2273-9.

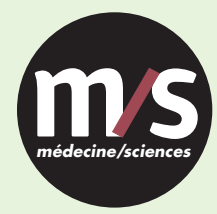

Tarifs d'abonnement $\mathrm{m} / \mathrm{s}-2013$

Abonnez-vous

à médecine/sciences
$>$ Grâce à $m / s$, vivez en direct les progrès des sciences biologiques et médicales

Bulletin d'abonnement page 702 dans ce numéro de $\mathrm{m} / \mathrm{s}$

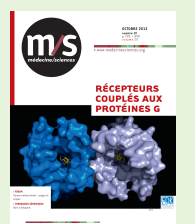

